\title{
DISPOSITIVO PARA AUXÍLIO DE DEFICIENTES VISUAIS UTILIZANDO SENSOR ULTRASSONICO
}

\author{
N.M.V. Alves*, A. J.A. Silva*, D. P. Salgado*, J.T. Faria*. \\ *Universidade Federal de Uberlândia, Uberlândia, Brasil \\ e-mail: nyallamary@gmail.com
}

\begin{abstract}
Resumo: Este artigo apresenta um protótipo desenvolvido para o auxílio de pessoas que apresentam deficiência visual, a fim de orientá-las sobre a distância em que sua mão se encontra de um objeto. O protótipo é composto por sensor ultrassônico, vibracall, buzzer e potenciômetro interligados entre si e conectados a um Arduino Pro Mini. Este último compreende a distância do sensor, colocado na mão do indivíduo e o alerta. A partir de distâncias pré-determinadas, o equipamento emite vibrações e sinais auditivos, que varia em intensidade e frequencia de acordo com a proximidade do objeto.
\end{abstract}

Palavras-chave: Deficiência Visual, Arduíno, vibracall, Ultrassom, buzzer.

\begin{abstract}
This paper presents a prototype developed to aid people with visual shortcoming in order to educate them about the distance that their hand is to an object. The prototype is composed of an ultrasonic sensor, vibrate, a buzzer and potentiometer interconnected and connected to a Arduino UNO, that comprehend the distance sensor setted in the hand of the individual and the alert. As from the predetermined distances, the equipment emits vibrations and audio signals, which become more intense, and frequently according to the proximity of the object.
\end{abstract}

Keywords: visual impairment, Arduino, vibrate, Ultrasound, buzzer..

\section{Introdução}

A deficiência é definida segundo a Organização Mundial da Saúde (OMS) como "problemas nas funções ou nas estruturas do corpo" [1,2]. A deficiência visual em especial compromete a segurança para a realização das atividades de vida diária [3]. Segundo dados, do Censo de 2010, existem no Brasil cerca 6,5 milhões de pessoas com deficiência visual, sendo 582 mil cegas e seis milhões com baixa visão [4]. É comum que pessoas que apresentam pequena porcentagem de visão, ou nenhuma, sofram acidentes enquanto se locomovem ou realizam ações cotidianas. Grande parte dos deficientes visuais é auxiliada somente por uso de bengalas, e se guiam de acordo com o toque da mesma no solo ou próxima a ele. Obstáculos que, por ventura, estejam em posições mais altas, são frequentemente despercebidos e desta forma, geram eventualidades.

Usualmente observa-se ainda, a dificuldade em estimar distancias, em virtude da localização imprecisa de um objeto. Inúmeros incidentes podem ocorrer em tarefas corriqueiras como pegar um copo, por exemplo, devido à dificuldade apresentada pelo indivíduo em determinar a extensão do movimento que deve se realizar para segurar um instrumento. O desenvolvimento do equipamento se deu, portanto, com a finalidade de auxiliar esses deficientes na precisão das distancias de objetos.

Sabe-se que demais capacidades sensoriais, como habilidades auditivas, podem vir a desenvolver-se com o déficit visual [3]. Com o propósito de atingir ainda mais pessoas, o dispositivo conta com alertas sonoros que permitem determinar se objetos ou obstáculos estão mais ou menos próximos.

Para compreender melhor acerca do desempenho do equipamento, o funcionamento de cada um dos componentes utilizados foi cuidadosamente analisado. São eles:

- Sensor ultrassônico é um elemento de caráter analógico que detecta massas sólidas e líquidas, sem necessidade de contato. É composto por dois canais: um como fonte de emissão de ondas e um como recepção de ondas. Operam através da emissão de uma radiação limpa e que não se sujeita a interferência de outras fontes que emitam radiações eletromagnéticas. Funcionam com a emissão de ondas, através do canal de emissão, que ao encontrar um obstáculo, são refletidas de volta ao sensor e captadas pelo canal de recepção, indicando a presença de um anteparo na sua zona de detecção. O comprimento de onda e a frequência de operação do sensor ultrassônico são, portanto, fatores determinantes da eficiência do mesmo para detecção dos objetos.

- buzzer é um componente eletrônico composto por duas camadas de metal e uma camada interna de cristal pizoelétrico, que recebe determinada fonte de energia e a traduz em frequência sonora. $\mathrm{O}$ estímulo do buzzer por um circuito faz com que este emita alertas auditivos em formas de beep, que podem ser contínuos ou não, de acordo com a programação inserida no circuito que o comanda.

- vibracall é um motor elétrico que responde a comandos do circuito inserido. Possui formato circular e é composto por placas metálicas e 
placa pizoelétrica que respondem a variação de tensão imposta sobre ele com vibrações. No equipamento, as vibrações ocorrem de acordo com a proximidade dos objetos detectados

- Potenciômetro é um componente eletrônico com capacidade de variar sua resistência interna, e desta forma, variar passagem de tensão e assim, parâmetros de controle. Foi utilizado a fim de variar o limiar de operação do sensor ultrassônico, aumentando ou diminuindo a distância de alerta.

- Arduino Pro Mini é micro controlador que engloba software e hardware, onde o software, programável externamente, interage diretamente com o hardware aplicado ao mesmo. Apresenta pinos de alimentação, de entradas e saídas, analógicas e digitais, modulação de pulso aplicada, botão de reinicialização, conexão com portas USB, entre outras funcionalidades.

Basicamente, o funcionamento do equipamento se dá através do sinal captado pelo sensor ultrassônico, determinado no potenciômetro, que é inserido no Arduino Pro Mini. Esse comanda, então, os componentes buzzer e vibracall, fazendo-os realizar atividades de acordo com os parâmetros estabelecidos na programação.

\section{Materiais e métodos}

O equipamento foi desenvolvido a partir dos seguintes elementos:

- Arduino Pro Mini;

- Sensor Ultrassom Arduino;

- Vibracall LG;

- Buzzer;

- Diodo Zener;

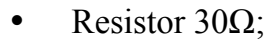

- Potenciômetro.

O sistema é alimentado com uma bateria externa, com tensão de $9 \mathrm{~V}$ e promove a interação entre os componentes acima dispostos da seguinte forma:

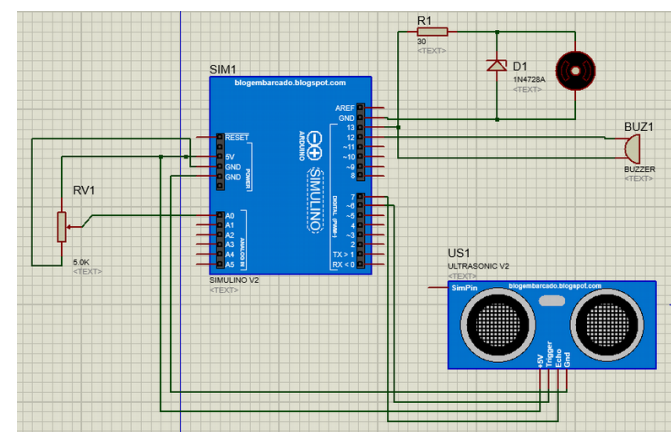

Figura 1. Circuito esquemático utilizado.

$\mathrm{Na}$ programação do Arduino foram definidos os limiares de atuação e as funções que deveriam ser realizadas de acordo com os limiares apresentados.
Foram criadas funções para serem desempenhadas de acordo com a resposta de proximidade dada pelo sensor de ultrassom. Os limiares foram definidos na faixa de $0,4 \mathrm{~m}$ e $0,05 \mathrm{~m}$. Entende-se que acima de 0,4 m, o indivíduo não corre algum tipo de risco ou não está suficiente perto de algum objeto para ser capaz de segurá-lo. Abaixo de $0,05 \mathrm{~m}$, o indivíduo já tem a convicção da ação que deseja realizar, pegando o objeto ou desviando do mesmo, e por isto, o sinal de alerta é interrompido. A comunicação serial foi configurada com 9600 BPS, ou seja, 9600 bits por segundo são recebidos. As leituras foram feitas a partir das entradas A3 (entrada analógica de número 3 , através do potenciômetro que define o limiar de operação. As saídas digitais 8 e 9, que também são saídas moduladoras de pulso, foram configuradas como saídas dos dispositivos de alerta, o vibracall e o buzzer, sendo a saída 9 conectada ao vibracall e a saída 8 , ao buzzer.

Desta forma, o esquema de funcionamento do equipamento se dá através da emissão de ondas ultrassonoras, que ao se depararem com um objeto com distância menor ou igual a $0,4 \mathrm{~m}$, retorna uma mensagem de vibração, em um ciclo de 0,5 segundos. Quando a distância entre equipamento fica entre $0,1 \mathrm{~m}$ e $0,05 \mathrm{~m}$, são emitidos sinais auditivos pelo buzzer, com ciclos de 0,5 segundos, além dos sinais vibratórios já emitidos.

Ao total, o equipamento mede $0,36 \mathrm{~m}^{2}$, com dimensões de $0,06 \mathrm{~m}$ de largura e $0,06 \mathrm{~m}$ de altura, e apresenta $0,03 \mathrm{~m}$ de profundidade. A pulseira, usada como suporte para o protótipo mede $0,2 \mathrm{~m}$ de comprimento, e sua colocação é feita por encaixe.

\section{Resultados}

Desenvolvido para auxílio de detecção de objetos e sinalização sobre a proximidade de obstáculos, o equipamento se mostrou eficiente. Foram realizados vários testes, em diversas ocasiões e com objetos de diferentes composições. Independente do estado do objeto, sólido como um copo, líquido como fluxo de água proveniente de uma torneira, ou granular, o sensor ultrassônico capta a presença e o circuito emite os sinais de alerta, dentro dos limiares impostos.

Foi testada também a continuidade dos alertas emitidos, uma vez que a mão do indivíduo esteja em contínuo movimento. E, novamente, independente da composição do objeto, os alertas foram intensificados em potência e frequência, de acordo com a maior proximidade.

\section{Discussão}

O uso de sensores ultrassônicos para a detecção de objetos seria de grande auxílio para deficientes visuais, considerando que o apoio comum para os mesmos é focado no ato de locomoção do corpo como um todo, através da caminhada. Com a utilização da bengala, o deficiente visual não tem percepção de anteparos que estejam posicionados acima dos membros inferiores, a não ser pelo tato. De qualquer forma, o tato expõe o 
deficiente visual a incidentes comuns, que podem ser evitados com a utilização do sensor ultrassônico. Além disso, o dispositivo se mostra uma ferramenta de ótimo custo-benefício em virtude de seu baixo custo.

As maiores dificuldades enfrentadas na execução e desenvolvimento do equipamento foram o tamanho e o peso da bateria externa, pela qual o circuito é alimentado. Além do acondicionamento do circuito em um suporte que fosse ao mesmo tempo eficiente e seguro para o protótipo, e fidedigno à variação do limiar. Observou-se ainda que, sendo o potenciômetro, um componente que apresenta grande impedância interna, a alimentação do circuito não se fez suficiente para que pudesse ser variada.

\section{Conclusão}

Ponderando os pontos conflitantes para o desempenho do protótipo, mesmo que sejam prejudiciais a aspectos estéticos e de utilização, o funcionamento do sistema não é comprometido. Desta forma, pode-se inferir que o mesmo é eficaz naquilo em que se propõe.

Considerando a hipótese de uma utilização real e contínua do equipamento, ele minimizaria as dificuldades táteis de deficientes visuais, facilitaria a realização de atividades cotidianas, evitaria acidentes e possui excelente custo-benefício ao usuário.

\section{Agradecimentos}

Os autores agradecem ao professor Doutor Adriano de Oliveira Andrade pelos ensinamentos acerca de interfaces entre homens e máquinas, para âmbitos da saúde, que tange à orientação para este projeto.

\section{Referências}

[1] Organização Mundial da Saúde. CIF: Classificação Internacional de funcionalidade, incapacidade e saúde. São Paulo: Edusp; 2003.

[2] Castro S.S., César C.L.G., Carandina L., Barros M.B.A., Alves C.G.P., Goldbaum M. “ Deficiência visual, auditiva e física: prevalência e fatores associados em estudo de base populacional. Caderno de Saúde Pública, Rio de Janeiro, 24(8): 1773-178. Agosto, 2008.

[3] Dias T.L.L., Pereira L. D. "Habilidade de localização e laterização sonora em deficientes visuais". São Paulo - SP. Revista Brasileira de Fonoaudiologia. 2008; 13(4): 352-6.

[4] ]Portal Brasil: Braile aumenta inclusão de cegos na s oc i edade. 2015 . Dis ponível e m: $<$ www.brasil.gov.br/cidadania-ejustiça/2015/01/braile-aumenta-inclusao-de-cegosna-sociedade. $>$.

[5] Sensores Ultrassônicos, Disponível em: $<$ www.automatizesensores.com.br/ultrasonicos.htm $>$
[6] WHO. World Report. World report on disability, n. WHO/NMH/VIP/11.01, p. 1-23, 2011. Disponível em: <www.who.int>.

[7]Datasheet, ATMEL 8-BIT Microcontroller with 4/8/16/32KBYTES in-system programmable flash d a $\mathrm{t}$ a $\mathrm{s}$ h e e $\mathrm{t}$. Disponível e m : $<$ www.atmel.com/images/Atmel-8271-8-bit-AVRMicrocontroller-ATmega48A-48PA-88A-88PA168A-168PA-328-328P_datasheet_Complete.pdf $>$. 